\title{
A Stability Result for the MCS Scheme Applied to 2D Convection-Diffusion Equations with Mixed Derivative
}

\author{
Chittaranjan Mishra \\ Department of Mathematics and Computer Science, University of Antwerp, \\ Middelheimlaan 1, 2020 Antwerp, Belgium
}

\begin{abstract}
This paper discusses the stability of the Modified Craig-Sneyd scheme when applied to two-dimensional convection-diffusion equations with mixed derivatives. A preliminary result is summarized that specially considers the size of the mixed derivative coefficients, which is very useful in practice.
\end{abstract}

Keywords: Options prices, convection-diffusion equation, finite difference methods, ADI splitting schemes, von Neumann stability PACS: $89.65 . \mathrm{Gh}, 02.70 . \mathrm{Bf}$

\section{INTRODUCTION}

In the contemporary literature, financial option prices are often modelled by time-dependent multidimensional partial differential equations (PDEs) with mixed derivatives. The presence of the mixed derivative terms, which arise from underlying correlated stochastic processes, forms an important characteristic of these PDEs. An analytical solution to the pricing PDE is often not available in a closed form, thus necessitating the PDE to be solved numerically. A special class of splitting schemes, of the alternating direction implicit (ADI) type, are popular among practitioners for this purpose, due to their computational advantage over common implicit schemes, e.g., Crank-Nicolson. ADI schemes, however, were not originally developed to handle mixed derivative terms.

In order to deal with PDEs with mixed derivative terms, In 't Hout \& Welfert [6] have recently formulated an ADI scheme known as the Modified Craig-Sneyd (MCS) scheme. The scheme has been applied successfully, e.g. in [2], to price options in the Heston frame-work and is one of the most prominent ADI schemes currently known. Stability of this scheme, being an important issue, was studied in [6] for pure-diffusion problems and in [4] for convectiondiffusion problems, where many positive results were obtained. These results, however, do not take into account the actual size of mixed derivative coefficients. We recently initiated research in this direction and obtained a variety of stability results for ADI schemes in $[3,5]$ that generalize the previously known results corresponding to pure-diffusion equations.

This paper investigates stability of the MCS scheme for time-dependent two-dimensional convection-diffusion equations, with an emphasis on the size of mixed derivative coefficients,

$$
\frac{\partial u}{\partial t}=c_{1} u_{x_{1}}+c_{2} u_{x_{2}}+d_{11} u_{x_{1} x_{1}}+\left(d_{12}+d_{21}\right) u_{x_{1} x_{2}}+d_{22} u_{x_{2} x_{2}}, \quad 0<x_{1}, x_{2}<1, \quad t \geq 0 .
$$

Here $c_{1}, c_{2}$ are given real numbers and $D=\left(d_{i j}\right)$ is a given symmetric positive semidefinite real matrix. For any given $\gamma \in[0,1]$, we consider in this paper the following condition on the diffusion matrix $D$,

$$
\left|d_{12}+d_{21}\right| \leq 2 \gamma \sqrt{d_{11} d_{22}} .
$$

The value $\gamma=0$ clearly yields the smallest possible mixed derivative coefficient, whereas $\gamma=1$ admits the largest possible, given that $D$ is positive semidefinite. In applications, it usually holds that $0<\gamma<1$.

Finite difference discretization of all spatial derivatives in (1), on a Cartesian grid, gives rise to a large system of ordinary differential equations (ODEs)

$$
U^{\prime}(t)=F(t, U(t)) \quad(t \geq 0), \quad U(0)=U_{0},
$$

with given vector-valued function $F$, given initial vector $U_{0}$ and unknown vectors $U(t)(t>0)$. We split the function $F$ into a sum

$$
F(t, v)=F_{0}(t, v)+F_{1}(t, v)+F_{2}(t, v)
$$


in such a way that $F_{0}$ corresponds to the discretized mixed derivative term and $F_{j}$ (for $j=1,2$ ) corresponds to all discretized spatial derivatives in the $j$-th direction. Define a temporal grid by $t_{n}=n \cdot \Delta t(n=0,1,2, \ldots)$ with given $\Delta t>0$. Then the MCS scheme defines approximations $U_{n}$ to $U\left(t_{n}\right)$ successively for $n=1,2, \ldots$ by

$$
\left\{\begin{array}{l}
Y_{0}=U_{n-1}+\Delta t F\left(t_{n-1}, U_{n-1}\right), \\
Y_{j}=Y_{j-1}+\theta \Delta t\left(F_{j}\left(t_{n}, Y_{j}\right)-F_{j}\left(t_{n-1}, U_{n-1}\right)\right), \quad j=1,2, \\
\widehat{Y}_{0}=Y_{0}+\theta \Delta t\left(F_{0}\left(t_{n}, Y_{2}\right)-F_{0}\left(t_{n-1}, U_{n-1}\right)\right), \\
\widetilde{Y}_{0}=\widehat{Y}_{0}+\left(\frac{1}{2}-\theta\right) \Delta t\left(F\left(t_{n}, Y_{2}\right)-F\left(t_{n-1}, U_{n-1}\right)\right), \\
\widetilde{Y}_{j}=\widetilde{Y}_{j-1}+\theta \Delta t\left(F_{j}\left(t_{n}, \widetilde{Y}_{j}\right)-F_{j}\left(t_{n-1}, U_{n-1}\right)\right), \quad j=1,2, \\
U_{n}=\widetilde{Y}_{2}
\end{array}\right.
$$

where $\theta>0$ is a given real parameter. The scheme (5) is known to have classical order of consistency two for any value of $\theta$. For $\theta=\frac{1}{2}$, it is identical to the Craig-Sneyd scheme proposed in [1]. A close look at the scheme reveals that the mixed derivative part, i.e., the $F_{0}$ term, is always treated explicitly, whereas $F_{j}(j=1,2)$ are treated implicitly. The scheme (5) has the well-known advantage of ADI schemes over standard implicit schemes in the sense that the systems of equations to be solved in each time-step are much easier to handle.

Consider the scalar test equation

$$
U^{\prime}(t)=\left(\lambda_{0}+\lambda_{1}+\lambda_{2}\right) U(t) \quad(t \geq 0)
$$

with complex constants $\lambda_{j}(0 \leq j \leq 2)$. Define $z_{j}=\Delta t \cdot \lambda_{j}(0 \leq j \leq 2)$ and denote

$$
z=z_{1}+z_{2} \text { and } p=\left(1-\theta z_{1}\right)\left(1-\theta z_{2}\right) .
$$

When applied to the test equation (6), the MCS scheme yields the scalar iteration

$$
U_{n}=S\left(z_{0}, z_{1}, z_{2}\right) U_{n-1}
$$

with

$$
S\left(z_{0}, z_{1}, z_{2}\right)=1+\frac{z_{0}+z}{p}+\theta \frac{z_{0}\left(z_{0}+z\right)}{p^{2}}+\left(\frac{1}{2}-\theta\right) \frac{\left(z_{0}+z\right)^{2}}{p^{2}} .
$$

In the von Neumann stability analysis of time-stepping schemes for (3), (4) the $\lambda_{j}$ are eigenvalues of $F_{j}(0 \leq j \leq 2)$. The iteration (7) is stable if

$$
\left|S\left(z_{0}, z_{1}, z_{2}\right)\right| \leq 1 .
$$

It can be shown that with respect to the standard finite difference discretization and the condition (2) on diffusion matrix, the scaled eigenvalues $z_{j}$ satisfy the property

$$
\left|z_{0}\right| \leq 2 \gamma \sqrt{\Re z_{1} \Re z_{2}}, \quad \Re z_{1} \leq 0, \quad \Re z_{2} \leq 0 .
$$

The proof of (10) follows directly from [7]. Given that the property (10) is satisfied for a given $\gamma \in[0,1]$, our interest is to investigate the values of the parameter $\theta$ for which the MCS scheme satisfies (9).

\section{THE MAIN RESULT}

It was shown in [4, Theorem 2.1] that if (10) holds and $\gamma=0$ (corresponding to no mixed derivative in (1)), then (9) is satisfied whenever $\theta \geq \frac{1}{4}$, whereas [4, Theorem 2.7] says that if (10) holds with $\gamma=1$, then (9) is satisfied whenever $\frac{1}{2} \leq \theta \leq 1$. It is worth noting that a smaller $\theta$ in the MCS scheme results in a smaller error constant and better damping properties, hence is more favorable. The following theorem forms a useful and interesting extension to the results reviewed above.

Theorem 1. Let $\gamma \in\left[0, \frac{1}{2}\right]$. Assume that $z_{j} \in \mathbf{C}(0 \leq j \leq 2)$ and (10) holds. Then $\left|S\left(z_{0}, z_{1}, z_{2}\right)\right| \leq 1$ whenever $\frac{1}{4} \leq \theta \leq 1$. 
For the sake of brevity, we give here a sketch of the proof. The complete proof will appear in a future paper.

Proof. For real $\varphi, r$, define

$$
f_{1}(\varphi, r)=\left|2 \theta+(1-\theta)\left(r e^{\mathbf{i} \varphi}-1\right)\right|
$$

and

$$
f_{2}(\varphi, r)=\left|8 \theta^{2}+4 \theta\left(r e^{\mathbf{i} \varphi}-1\right)+(1-2 \theta)\left(r e^{\mathbf{i} \varphi}-1\right)^{2}\right| .
$$

For any given $\gamma \in\left[0, \frac{1}{2}\right]$, denote

$$
f(\varphi, r)=\frac{\gamma^{2}(1-r)^{2}+2 \gamma(1-r) f_{1}(\varphi, r)+f_{2}(\varphi, r)}{8 \theta^{2}} .
$$

Analogous to the proof of [4, Theorem 2.7], we have

$$
\left|S\left(z_{0}, z_{1}, z_{2}\right)\right| \leq \max \{f(\varphi, r): 0 \leq \varphi \leq 2 \pi, 0 \leq r \leq 1\} .
$$

Now it can be shown that for $\varphi \in[0,2 \pi]$ and $r \in[0,1]$ :

$$
f_{1}(\varphi, r) \leq|1-3 \theta|+r(1-\theta)
$$

and

$$
f_{2}(\varphi, r) \leq 2 \theta\left(4 \theta-1+r^{2}\right)
$$

whenever $\frac{1}{4} \leq \theta \leq \frac{1}{2}$. The derivation of (12) is straightforward, whereas that of (13) is more involved. With these estimates at hand, we prove the main theorem by considering three separate domains of $\theta$. Let $\varphi \in[0,2 \pi]$ and $r \in[0,1]$.

$$
\begin{aligned}
& \text { For } \frac{1}{4} \leq \theta \leq \frac{1}{3} \text { : } \\
& f(\varphi, r) \leq \frac{1}{8 \theta^{2}}\left\{\left(\frac{1-r}{2}\right)^{2}+(1-r)(1-3 \theta+r(1-\theta))+2 \theta\left(4 \theta-1+r^{2}\right)\right\} \\
& =1+\frac{1}{8 \theta^{2}}\left(\theta-\frac{1}{4}\right)\left(3 r^{2}+2 r-5\right) \\
& \leq 1 \text {. } \\
& f(\varphi, r) \leq \frac{1}{8 \theta^{2}}\left\{\left(\frac{1-r}{2}\right)^{2}+(1-r)(3 \theta-1+r(1-\theta))+2 \theta\left(4 \theta-1+r^{2}\right)\right\} \\
& =1+\frac{r-1}{8 \theta^{2}}\left\{\left(\theta-\frac{1}{4}\right)(3 r-1)+\frac{1}{2}\right\} \\
& \leq 1 \text {. }
\end{aligned}
$$

For $\frac{1}{2} \leq \theta \leq 1:$

As a direct consequence of [4, Theorem 2.7], we have

$$
f(\varphi, r) \leq 1
$$




\section{CONCLUSION}

In financial applications $\gamma \in\left[0, \frac{1}{2}\right]$ represents the fact that the underlying stochastic processes have a maximum correlation of \pm 50 percent. If we consider the two-dimensional convection-diffusion equation (1), (2) with $\gamma=\frac{1}{2}$, then the MCS scheme (5), when applied to the semidiscretized, splitted system (4), is unconditionally stable, in the von Neumann sense, whenever $\frac{1}{4} \leq \theta \leq 1$.

\section{ACKNOWLEDGMENTS}

I am grateful to Karel in 't Hout for his invaluable suggestions on different issues in the article.

\section{REFERENCES}

1. I. J. Craig \& A. D. Sneyd, An alternating-direction implicit scheme for parabolic equations with mixed derivatives, Comput. Math. Applic. 16 (1988) 341-350.

2. K. J. in 't Hout \& S. Foulon, ADI finite difference schemes for option pricing in the Heston model with correlation, Int. J. Numer. Anal. Mod. 7 (2010) 303-320.

3. K. J. in 't Hout \& C. Mishra, Stability of ADI schemes for multidimensional diffusion equations with mixed derivative terms, Submitted (2012).

4. K. J. in 't Hout \& C. Mishra, Stability of the modified Craig-Sneyd scheme for two-dimensional convection-diffusion equations with mixed derivative term, Math. Comp. Simul. 81 (2011) 2540-2548.

5. K. J. in 't Hout \& C. Mishra, A stability result for the modified Craig-Sneyd scheme applied to $2 D$ and $3 D$ pure diffusion equations, In: Numerical Analysis and Applied Mathematics, eds. T. E. Simos et. al., AIP Conf. Proc. 1281 (2010) $2029-2032$.

6. K. J. in 't Hout \& B. D. Welfert, Unconditional stability of second-order ADI schemes applied to multi-dimensional diffusion equations with mixed derivative terms, Appl. Numer. Math. 59 (2009) 677-692.

7. K. J. in 't Hout \& B. D. Welfert, Stability of ADI schemes applied to convection-diffusion equations with mixed derivative terms, Appl. Numer. Math. 57 (2007) 19-35. 
Copyright of AIP Conference Proceedings is the property of American Institute of Physics and its content may not be copied or emailed to multiple sites or posted to a listserv without the copyright holder's express written permission. However, users may print, download, or email articles for individual use. 